\title{
Stereological and Biochemical Analysis of the Urethral Edges in Patients Submitted to End-to-End Anastomosis for Bulbar Urethral Stricture
}

Joao P. M. de Carvalho, Andre G. Cavalcanti, Waldemar S. Costa, Luiz E. M. Cardoso, Francisco J. B. Sampaio, Luciano A. Favorito

Urogenital Research Unit, State University of Rio de Janeiro (JPMC, AGC, WSC, LEMC, LAF, FJBS), Rio de Janeiro, Brazil and Section of Urology, Souza Aguiar Municipal Hospital (AGC, LAF), Rio de Janeiro, Brazil

\section{ABSTRACT}

Purpose: To study the morphologic alterations in the proximal and distal urethral edges from patients submitted to end-to-end bulbar urethroplasty.

Materials and Methods: We analyzed 12 patients submitted to anastomotic urethroplasty to treat bulbar strictures less than $2.0 \mathrm{~cm}$ in length. After excision of the fibrotic segment to a $28 \mathrm{Fr}$ urethral caliber, we obtained biopsies from the spongious tissue of the free edges (proximal: PROX and distal: DIST). Controls included normal bulbar urethras obtained from autopsies of 10 age matched individuals. The samples were histologically processed for smooth muscle cells (SMC), elastic system fibers and collagen. Stereological analysis was performed to determine the volumetric density (Vv) of each element. Also, a biochemical analysis was performed to quantify the total collagen content.

Results: $\mathrm{Vv}$ of SMC was reduced in PROX $(31.48 \pm 7.01 \mathrm{p}<0.05)$ and similar in DIST when compared to controls $(55.65 \pm 9.60 \%)$ with no statistical difference. Elastic fibers were increased in PROX $(25.70 \pm 3.21 \% ; p<0.05)$ and were similar to controls in DIST $(15.87 \pm 4.26 \%)$. Total collagen concentration in PROX $(46.39 \pm 8.20 \mu \mathrm{g} / \mathrm{mg})$, and DIST $(47.96 \pm 9.42 \mu \mathrm{g} / \mathrm{mg})$ did not differ from controls $(48.85 \pm 6.91 \mu \mathrm{g} / \mathrm{mg})$. Type III collagen was similarly present in all samples.

Conclusions: After excision of the stenotic segment to a caliber of $28 \mathrm{Fr}$, the exposed and macroscopically normal urethral edges may present altered amounts of elastic fibers and SMC, but are free from fibrotic tissue. When excising the peri-stenotic tissue, the surgeon should be more careful in the proximal end, which is the most altered.

\section{ARTICLE INFO}

\section{Key words:}

Urethra, Stricture, Urethroplasty, Extracellular matrix, Smooth muscle

Int Braz J Urol. 2012; 38: 674-81

Submitted for publication: January 16, 2012

Accepted after revision:

March 28, 2012

\section{INTRODUCTION}

Male urethral stricture is characterized by a fibrous process, which leads to the development of a low compliance tissue, associated with reduction of the luminal diameter (1). Previous studies documented a negative correlation between urethral diameter and both the AUA urinary symptoms score and maximum flow rate (2).

These alterations can severely worse the voiding pattern and consequently compromise patient's quality of life (3). The extracellular matrix (ECM), notably its fibrous components such as collagen and elastic fibers, participates 
actively in the tissue organization and in urethral physiopathology $(4,5)$.

Structural changes observed in urethral stenosis were first described by Singh (1), who through light microscopy, observed that the collagen was increased and less organized. They also noted a replacement of muscle cells by dense collagen.

In urethral stenosis treatment, it is important to take into account its localization, extension and degree of fibrosis of the corpus spongiosum (6). The endoscopic treatment of stenosis through the incision of the fibrotic tissue, with its posterior reepithelization, can be an option when the stenotic segment is short and has a limited degree of fibrosis (7).

Surgical treatment should preferentially be done by an anastomotic urethroplasty with complete resection of the fibrous tissue. The anastomosis should be done with macroscopically wealthy edges. This technique is limited to stenotic segments not greater than $2.0 \mathrm{~cm}$. In more extended strictures, substitution of the fibrous tissue by grafts is recommended (8). A previous study characterized the bulbar urethra stenosis and its fibrous tissue (9); however, there is little data on the surgically exposed edges. Indeed, it is noteworthy that investigations on this disease have concentrated on the fibrotic tissue $(5,9)$.

A recent study has evaluated the surgically exposed urethral ends, but only qualitative histological techniques were used (10). This fact, together with other technical limitations, may render these results less reliable. Thus, knowledge about the exposed urethral ends, whose quality may affect the surgical success of the anastomotic urethroplasty, is still unsettled.

Therefore, the aim of the present study was to perform a stereological and biochemical analysis of the urethral edges in patients undergoing end-to-end anastomotic bulbar urethroplasty for stricture disease.

\section{MATERIALS AND METHODS}

The present work was approved by the Bio-Ethics Committee of our institution. This study was carried out in accordance with the ethical standards of the responsible institutional committee on human experimentation. From May 2006 to August 2010, we analyzed 12 patients aged 13 to 43 years (mean $=32$ ) who had been submitted to end-to-end bulbar urethroplasty. The stenotic segment was shorter than 2.0 $\mathrm{cm}$ in all patients and was estimated by urethrocystogram and confirmed during surgery.

The etiology of the stenosis was idiopathic in 5 patients, inflammatory in 5 and traumatic in 2. All patients were submitted to uroflowmetry study and presented a maximum flow rate under $15 \mathrm{~mL} / \mathrm{s}$ (mean $5.3 \mathrm{~mL} / \mathrm{s}$ ). No patient was diverted prior the surgery. The proximal (PROX) and distal (DIST) extremities of the stenosis were excised by the usual technique (11) and by the same surgeon. The macroscopically fibrotic tissue was completely removed until a $28 \mathrm{Fr}$ dilator could pass through the luminal openings of both urethral edges. If the urethral edges thus exposed were macroscopically healthy, based on the criteria of Mundy (12), but the luminal openings were not large enough for a 28Fr dilator, approximately 2-mm circular slices were consecutively sectioned from the free ends until the desired diameter was obtained. Afterwards, a biopsy was obtained from these edges.

The control group comprised macroscopically normal bulbar urethras obtained during autopsy of 10 individuals (mean age 29 years) who died of causes not related to the urinary system or to pelvic trauma.

\section{Histology and Stereology}

Tissue specimens were fixed in 10\% buffered formalin and routinely processed for paraffin embedding. Sections of 5 - $\mu \mathrm{m}$ were obtained and stained with: (a) Masson Thricome's to label smooth muscle cells, (b) Weigert's resorcinfuchsin with previous oxidation to stain elastic system fibers, and (c) Picrosirius red under polarized light to detect differences in overall collagen organization.

Also, we performed immunohistochemical analysis to specifically detect collagen typeIII. The specificity of the Weigert's method was confirmed by immunolabeling with an anti-elastin antibody (monoclonal, E 4013, Sigma, Saint 
Louis, MO, USA), and that of the smooth muscle staining by immunolabeling with an anti-smooth muscle alpha actin (Zymed Laboratories, 08-0106 predilute antibody).

Morphological data was quantified using stereological methods. For each Individual and for each histological staining technique, 5 sections of each edge (PROX, DIST, Control) were obtained, and for each section, 5 fields were analyzed. All images were photographed with a digital camera directly coupled to the microscope at a final magnification of X200 to SMC and X400 for Elastic Fibers. The volumetric density (Vv) of histological structures was then evaluated by superimposing the 100 point grid test system on the digital images, using the Image $\mathrm{J}$ program (NIH Image ${ }^{\circledR}$ ), (13), Figure-1A.

\section{Biochemical Analysis}

Immediately after excision during surgery or autopsy, urethral tissue samples were fixed in cold acetone for 24 hours at $4^{\circ} \mathrm{C}$. The samples were then finely minced and submitted to two changes of 24 hours each in $40 \mathrm{~mL}$ of chloroform/ methanol $(2: 1, \mathrm{v} / \mathrm{v})$ at room temperature. The solvent was then decanted, and after incubation at $60^{\circ} \mathrm{C}$ for 30 minutes, a preparation of dry and defatted urethral tissue was obtained and weighed.

The concentration of total collagen in the urethral tissue was determined by a colorimetric hydroxyproline assay. Thus, 5 to $14 \mathrm{mg}$ of dry, defatted urethral tissue were hydrolyzed in $6 \mathrm{~N} \mathrm{HCl}$ for 18 hours at $118^{\circ} \mathrm{C}$ as previously described (14).

The assay was then carried out in the neutralized hydrolyzates using a chloramin $\mathrm{T}$ method (15). Results were expressed as micrograms of hydroxyproline per milligram of dry, defatted tissue.

\section{Statistics}

Statistical procedures followed Sokal and Rohlf (16). Variations among the three groups for each quantitative parameter were analyzed by one-way ANOVA. When significant differences were detected, pairwise planned comparisons using the Bonferroni method were done between the control group and either the PROX or the
DIST group. All numerical results are given as means \pm standard deviations (SD), and statistical significance was considered when $\mathrm{p}<0.05$.

\section{RESULTS}

The quantitative analysis showed a decrease in the volumetric density (Vv) of smooth muscle cells in PROX (43.40\%) edges, when compared to controls $(\mathrm{p}<0.05)$, with no significant difference in the DIST edge (Figures 1B, 1C and 1D and Table).

The elastic system fibers quantification showed a $44.40 \%$ increase in the Vv in the PROX edges, when compared to controls $(\mathrm{p}<0.05)$, while in the DIST edges the difference was not significant (Figure-2 and Table).

The Picrosirius Red stain showed a prevalence of greenish color in PROX edges whereas in the DIST edges there was a prevalence of a red/ yellowish color, similar to controls. The imunohistochemical analysis revealed the presence of similar amounts of type III collagen in all samples (Figure-3).

Total collagen concentration in controls $(48.85 \pm 6.91 \mu \mathrm{g} / \mathrm{mg})$, and in samples from the PROX $(46.39 \pm 8.20 \mu \mathrm{g} / \mathrm{mg})$ and DIST (47.96 \pm 9.42 $\mu \mathrm{g} / \mathrm{mg}$ ) edges did not differ significantly. Thus, the exposed edges of the two urethral ends after excision of the stenotic segment had similar collagen content and were free from fibrotic tissue.

\section{DISCUSSION}

In the best of our knowledge, this is the first study to perform a stereological and biochemical analysis on the urethral edges (proximal and distal) that will be anastomosed after resection of the stenosis in an end-to-end bulbar urethroplasty.

In the present work, we have found a decrease in the $\mathrm{Vv}$ of smooth muscle cells and an increase in the $\mathrm{Vv}$ of elastic fibers, both of which were more intense in the proximal urethral edge. Probably this happens since the proximal urethra is submitted to a much higher hydrostatic pressure, which can cause an increase in fibroblast activity (17). On the other hand, the urethra distally to 
Figure 1 - Smooth muscle cells (SMC) distribution in urethral samples. A) Photomicrography of a control urethra showing the morphometric analysis. Quantification of smooth muscular cells using the software Image J Test grid. Masson's trichrome X200. B) Smooth muscle cells (SMC) distribution in control group. Smooth muscle cells (SMC) distribution in proximal edge of stenotic group and D) Smooth muscle cells (SMC) distribution in distal edge of stenotic group. Note marked decrease of SMC(red) in the proximal edges and less marked decrease in the distal edge, when compared to controls (differences statistically significant). Masson's trichrome, X200.
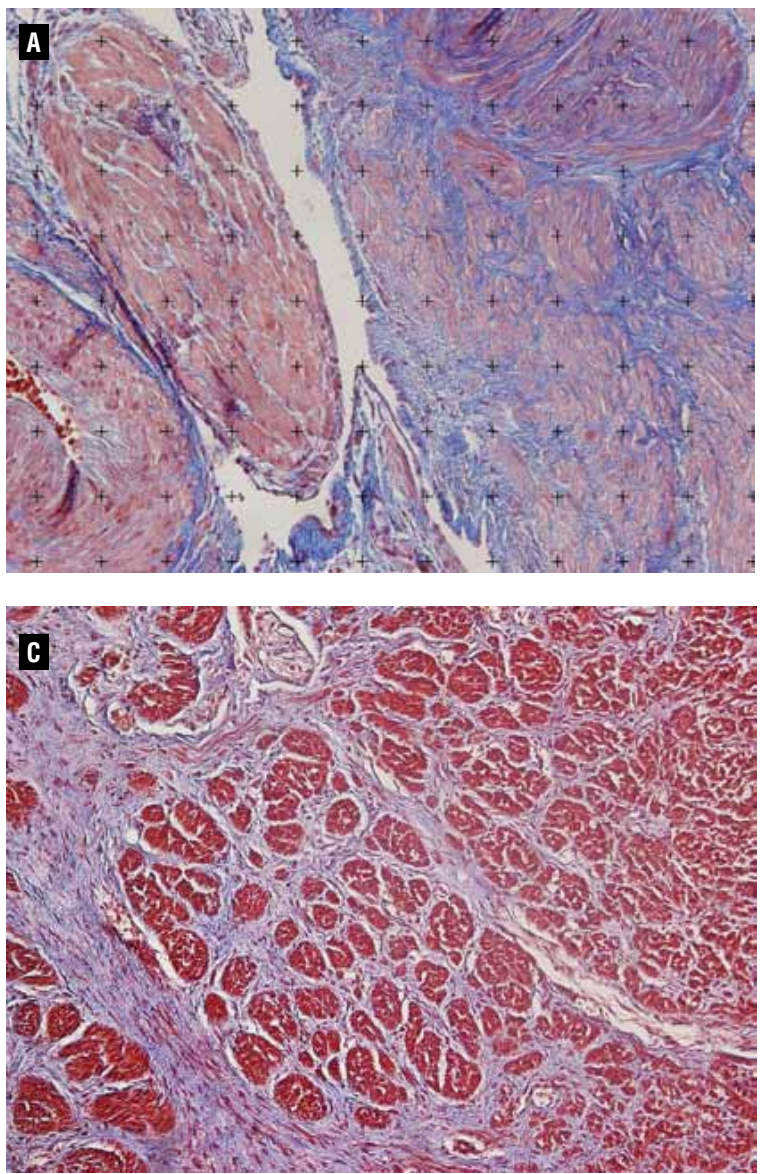
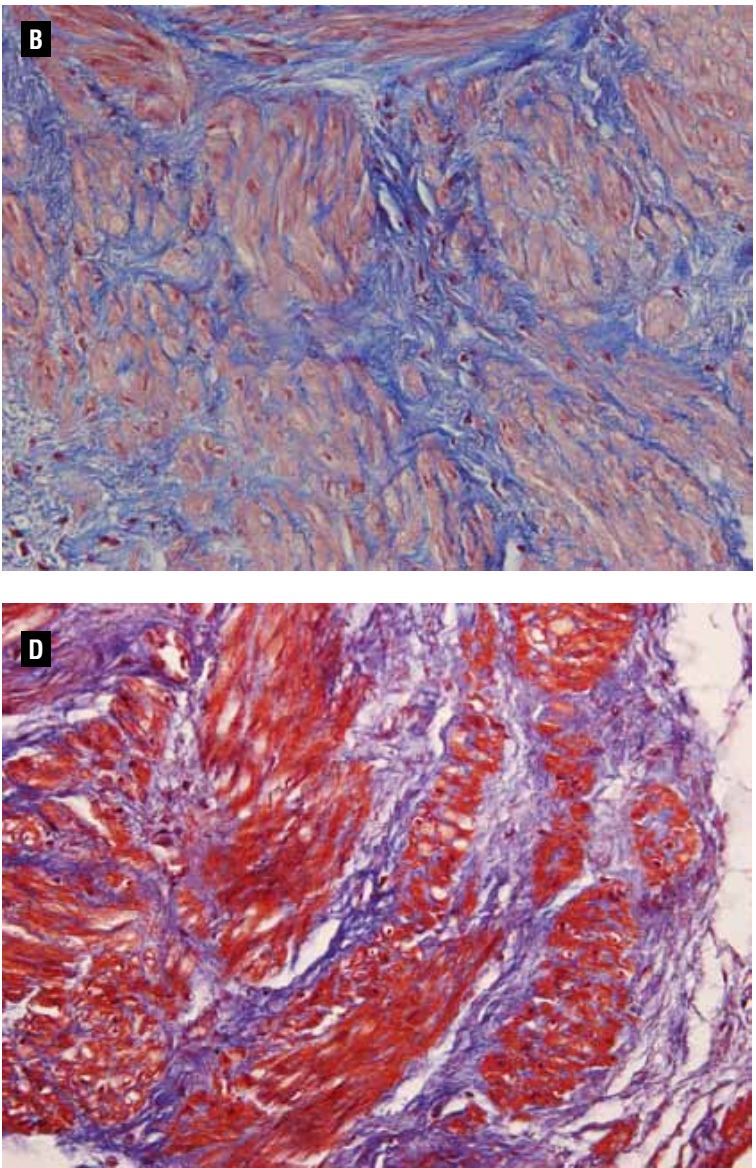

Table 1 - Stereological analysis of smooth muscle and elastic system fibers of urethral edges.

\begin{tabular}{lll}
\hline & Mean $(\%)$ & SD $( \pm)$ \\
\hline SM Controls & 55.64 & 9.60 \\
SM Prox & 31.48 & 7.01 \\
SM Dist & 50.97 & 10.27 \\
EF Controls & 17.79 & 2.82 \\
EF Prox & 25.70 & 3.21 \\
EF Dist & 15.87 & 4.26 \\
\hline
\end{tabular}

$\mathrm{SM}=$ smooth muscle; PROX = proximal urethral edges in stenotic group; DIST = distal urethral edges in stenotic group; $\mathrm{EF}=$ elastic fibers. $\mathrm{P}<$ 0.01 when comparing SM PROX with SM Controls. $P<0.01$ when comparing EF PROX with EF controls. 
Figure 2 - Elastic system fibers distribution in urethral samples from Controls (A), Proximal edge of stenotic group (B) and Distal edge of stenotic group (C). Note marked increase of elastic fibers in the proximal edge and similar content in the distal edge, when compared to controls. Weigert's Resorcin-Fuchsin, X400.
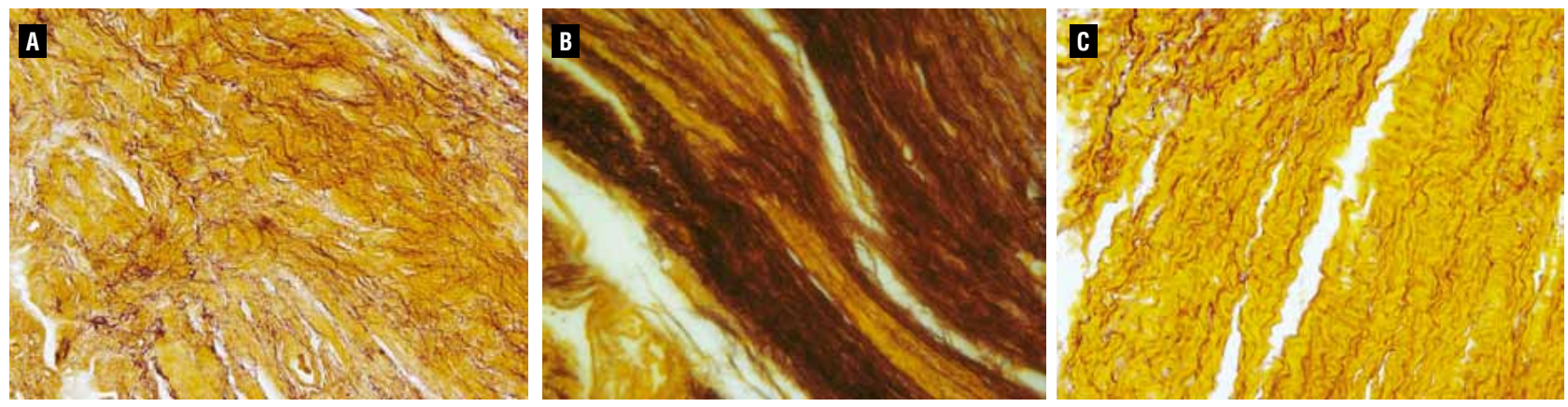

Figure 3 - Imunohistochemical staining for Type III collagen from Control group (A), Proximal edge of stenotic group (B) and Distal edge of stenotic group (C), showing a similar distribution in all groups (Immunolabelling, X200).
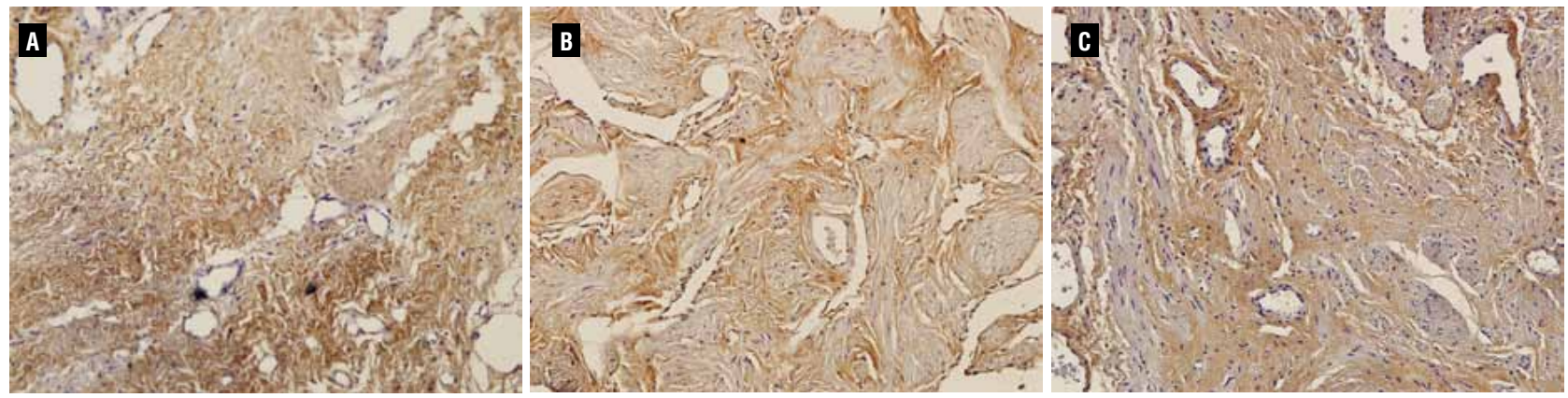

the stenosis is protected from high pressure and it could explain the less pronounced alterations.

It has been previously demonstrated that the lesions of nerves that carries the nitric oxide synthase (nNOS), in the cavernous body, leads to a fibrotic process associated to smooth muscle degeneration (18). A previous study of Cavalcanti et al. (19) documented an association of urethral stricture with alteration in nNOS imunorreactivity, even in small amounts of spongiofibrosis.

Based on these observations we could speculate that the smooth muscle reduction should be the primary event in urethral stenosis and fibrotic tissue formation. This event is intense in PROX margin, probably correlated to a hydrodistension process, which could also explain the structural differences between the urethral ends after excision of the stenotic area.

In the urethra distal to the stenosis, the tissue would be protected from higher pressure, and this could explain the less pronounced alterations at the distal edge, as commented previously. It is important to point out that none of the patients had cistostomy tubes (not diverted) prior surgery, and therefore, the patients had to void under high pressure due to the obstructive process.

Bastos (20) previously described the elastic system's fibers distribution in male fetal urethra and demonstrated that the concentration of elastic fibers in the spongy urethra increases significantly with age and this high concentration of elastic fibers may partially explain its high extensibility. Also, the elastic fibers concentration has a progressive increase during the fetal period gestation in a linear fashion, and continues to increase to the adulthood, which implies functional adaptation of the fetal male urethra.

In the present study, we observed an increase in elastic fibers volumetric density in 
the proximal margin when compared to control group. Thus, increased synthesis of elastic fibers may be associated with excessive distension of an organ, as it also occurs during bladder outlet obstruction (21,22). In our analysis, the area once before occupied by smooth muscle cells, has been replaced by elastic fibers, suggesting, once more, the hydrodistension process suffered by the proximal margin.

When using the fibrotic resection to a 28Fr luminal caliber, the biochemical analysis demonstrated that all fibrotic tissue was satisfactorily excised, as collagen concentrations were similar to control group. These results show that the surgical technique described in the present study guaranteed complete resection of the fibrotic segment.

It has been variously shown that a shift towards greenish color in the Picrosirius polarization method is associated with less organized and/or degraded collagen (23). This color change may also occur in earlier phases of the remodeling and repair of connective tissues, when the synthesis of collagen type III is enhanced (24). In fact, a green shift in the staining pattern produced by the Picrosirius-polarization method is more related to the organization or packing state of collagen fibrils in the tissue (24), and therefore it is not necessarily an indicative of the presence of collagen type III.

To test between these possibilities in our samples, we immunostained urethral sections with an anti-type III collagen antibody, which showed that the intensity and distribution of the labeling was similar in all three groups. Additionally, the biochemical assay indicated equal amounts of total collagen in the urethral edges.

Thus, and based on these findings, the results of the Picrosirius-polarization method suggest that collagen matrix at the proximal urethral edge is disrupted or degraded, rather than fibrotic, which is consistent with a higher hydrostatic pressure proximal to the stenotic segment. It should be mentioned that these results are in stark contrast with a recent report that used this same surgical procedure (10). In that study by Da Silva (10), urethral samples were stained with the Picrosiriuspolarization method, and based solely on the gen- erated color patterns, the results were expressed as a ratio of collagen types I and III.

Such a result implies that colors were quantified, although no information was provided on how this was done. Further, in their description of the results, the authors of that study limited themselves to stating that the ratio was altered at the proximal urethral edges. This again is odd because, insofar as putative collagen types, or ratios thereof, were quantitated, one could tell whether their amounts were increased or decreased.

Although we did not directly assess inflammatory cells at the urethral edges, the fact the collagen type III remained unchanged in samples from stricture patients suggests that there is no major inflammatory reaction in the tissue. This is also at variance with what was found by Da Silva (10), and it might be explained by differences in the surgical technique.

For example, after excision of the stenotic segment, the criteria normally used to consider the exposed urethral edges as suitable for anastomosis are their macroscopic aspect $(10,12)$. However, in our evaluation of tissue status, a healthy edge had in addition a normal luminal opening, as determined by the passage of a $28 \mathrm{Fr}$ dilator.

This approach may set the position of excision farther from the stenotic area, so that the exposed edges would be less altered. A healthier urethral end is likely to increase the success rate of the surgical anastomosis (25). However, because we do not have follow-up data for our patients, we cannot ascertain whether our particular technique improved the long-term outcome of the anastomosis.

Da Silva et al. (10) claimed to have followed their patients, yet no data on this regard was reported.

The study of tissue components in anastomotic margins should improve our understanding of the physiopathology process in urethral stricture and can be applied directly to the development of new therapeutic options.

In conclusion, our results have shown that, after excision of the stenotic segment to a luminal caliber of $28 \mathrm{Fr}$, the exposed and macroscopically proximal edge may present altered 
amounts of elastic fibers and SMC, as well as structural modifications in collagen, but are free from fibrotic and overtly inflammatory tissue.

These alterations are consistent with a higher hydrostatic pressure in the proximal urethra. All histological quantitative and qualitative findings of the present research indicate that when excising the peri-stenotic tissue, the surgeon should be more careful in the proximal end.

\section{ACKNOWLEDGEMENTS}

This study received institutional review committee approval.

Supported by grants from the National Council for Research Support (CNPq) and Rio de Janeiro Foundation for Research Support (FAPERJ).

\section{ABBREVIATIONS}

SMC $=$ smooth muscle cells

PROX = proximal urethral edge

DIST $=$ distal urethral edge

Vv = volumetric density

\section{CONFLICT OF INTEREST}

None declared.

\section{REFERENCES}

1. Singh M, Scott TM: The ultrastructure of human male urethral stricture. Br J Urol. 1975; 47: 871-6.

2. Heyns CF, Marais DC: Prospective evaluation of the American Urological Association symptom index and peak urinary flow rate for the followup of men with known urethral stricture disease. J Urol. 2002; 168: 2051-4.

3. McAninch JW, Armenakas NA: Acute Anterior Urethral Injuries: Diagnosis and Initial Management. In: McAninch JW (ed.), Traumatic and Reconstructive Urology. Philadelphia, WB Saunders Co. 1996; p. 543.

4. Baskin LS, Constantinescu SC, Howard PS, McAninch JW, Ewalt $\mathrm{DH}$, Duckett JW, et al.: Biochemical characterization and quantitation of the collagenous components of urethral stricture tissue. J Urol. 1993; 150: 642-7.
5. Da-Silva EA, Sampaio FJ, Dornas MC, Damiao R, Cardoso LE: Extracellular matrix changes in urethral stricture disease. J Urol. 2002; 168: 805-7.

6. Barbagli G, Palminteri E, Guazzoni G, Montorsi F, Turini D, Lazzeri M: Bulbar urethroplasty using buccal mucosa grafts placed on the ventral, dorsal or lateral surface of the urethra: are results affected by the surgical technique? J Urol. 2005; 174: 955-7; discussion 957-8.

7. Heyns CF, Steenkamp JW, De Kock ML, Whitaker P: Treatment of male urethral strictures: is repeated dilation or internal urethrotomy useful? J Urol. 1998; 160: 356-8.

8. Santucci RA, Joyce GF, Wise M: Male urethral stricture disease. J Urol. 2007; 177: 1667-74.

9. Cavalcanti AG, Costa WS, Baskin LS, McAninch JA, Sampaio FJ: A morphometric analysis of bulbar urethral strictures. BJU Int. 2007; 100: 397-402.

10. Da Silva EA, Schiavini JL, Santos JB, Damião R: Histological characterization of the urethral edges in patients who underwent bulbar anastomotic urethroplasty. J Urol. 2008; 180: 2042-6.

11. Jordan GH: Reconstruction for Urethral Stricture. In: Atlas of Urologic Clinics of North America, vol 5, no 1. Philadelphia, WB Sounders Co. 1997.

12. Mundy AR: Anastomotic urethroplasty. BJU Int. 2005; 96: $921-$ 44.

13. Chagas MA, Babinski MA, Costa WS, Sampaio FJ: Stromal and acinar components of the transition zone in normal and hyperplastic human prostate. BJU Int. 2002; 89: 699-702.

14. Cabral CA, Sampaio FJ, Cardoso LE: Analysis of the modifications in the composition of bladder glycosaminoglycan and collagen as a consequence of changes in sex hormones associated with puberty or oophorectomy in female rats. J Urol. 2003; 170 : 2512-6.

15. Bergman I, Loxley R: Two improved and simplified methods for the spectrophotometric determination of hydroxyproline. Anal Biochem. 1963; 35: 1961-5.

16. Sokal RR, Rohlf FJ: Biometry, ed. 3. New York, WH Freeman. 1995.

17. Junker JP, Kratz C, Tollbäck A, Kratz G: Mechanical tension stimulates the transdifferentiation of fibroblasts into myofibroblasts in human burn scars. Burns. 2008; 34: 942-6.

18. Squadrito GL, Pryor WA: Oxidative chemistry of nitric oxide: the roles of superoxide, peroxynitrite, and carbon dioxide. Free Radic Biol Med. 1998; 25: 392-403.

19. Cavalcanti AG, Yucel S, Deng DY, McAninch JW, Baskin LS: The distribution of neuronal and inducible nitric oxide synthase in urethral stricture formation. J Urol. 2004; 171: 1943-7.

20. Bastos AL, Silva EA, Silva Costa W, Sampaio FJ: The concentration of elastic fibres in the male urethra during human fetal development. BJU Int. 2004; 94: 620-3

21. Cortivo R, Pagano F, Passerini G, Abatangelo G, Castellani I: Elastin and collagen in the normal and obstructed urinary bladder. $\mathrm{Br}$ J Urol. 1981; 53: 134-7. 
22. Gosling JA: Modification of bladder structure in response to outflow obstruction and ageing. Eur Urol. 1997; 32(Suppl 1): 9-14.

23. Ushiki T: Collagen fibers, reticular fibers and elastic fibers. A comprehensive understanding from a morphological viewpoint. Arch Histol Cytol. 2002; 65: 109-26.
24. Hirshberg A, Lib M, Kozlovsky A, Kaplan I: The influence of inflammation on the polarization colors of collagen fibers in the wall of odontogenic keratocyst. Oral Oncol. 2007; 43: 278-82.

25. Santucci RA, Mario LA, McAninch JW: Anastomotic urethroplasty for bulbar urethral stricture: analysis of 168 patients. J Urol. 2002; 167: 1715-9.

Correspondence address: Dr. Luciano A. Favorito Av. 28 de Setembro, 87, Fundos, FCM, Terreo

Urogenital Research Unit - UERJ 20551-030, Rio de Janeiro, RJ, Brazil Fax: + 5521 2587-6499 E-mail: lufavorito@yahoo.com.br 